\title{
The ATLAS Trigger in 2017 and 2018: Developments and Performance
}

\author{
Daniele Zanzi* on behalf of the ATLAS Collaboration \\ CERN \\ E-mail: daniele.zanzi@cern.ch
}

\begin{abstract}
The ATLAS Trigger system has been operating successfully during LHC Run-2, between 2015 and 2018. Its excellent performance has been vital for the ATLAS physics program, selecting interesting collision events for a wide variety of physics signatures with high efficiency. This presentation gives a review of the ATLAS trigger system in 2017 and 2018, covering briefly changes compared to 2016, operational aspects and encountered constraints. Focus is put on the new event-topology-based selections at the Level-1 trigger and on the trigger performance at high pile-up seen in 2017 and 2018.
\end{abstract}

ICHEP 2018, XXXIX International Conference on High Energy Physics

4-11 July 2018

Seoul, South Korea

${ }^{*}$ Speaker. 
The trigger system [1] of the ATLAS detector [2] at the LHC is responsible for the real-time selection of the subset of collisions to be recorded and analysed offline. For Run-2, the ATLAS trigger system was significantly improved compared to Run-1 in order to cope with the higher event rates and with the large number $(\mu)$ of simultaneous proton-proton interactions (pile-up). It has been operating successfully during the LHC Run-2, from 2015 to 2018, collecting collision events for a wide variety of physics signatures with high efficiency.

This presentation focuses on the ATLAS trigger system in 2017 and 2018, when the LHC delivered collisions both at record high instantaneous luminosities, up to $2 \times 10^{34} \mathrm{~cm}^{-2} \mathrm{~s}^{-1}$, and at record high pile-up, up to $\mu=60$. The ATLAS trigger system successfully adapted to these challenging LHC running conditions thanks to new hardware and improvements in software.

The Run-2 ATLAS trigger system [1] comprises two levels. The first-level trigger (Level 1, L1) is implemented in hardware and uses a subset of the information from the calorimeter and muon detectors to reduce the accepted rate to a design maximum of $100 \mathrm{kHz}$. It has a fixed $2.5 \mu$ s latency after which events are rejected or passed to the High-Level trigger (HLT). The HLT is implemented in software in a farm with about 40 to 50 thousands processing units. It reconstructs features using full-granularity detector information either in the Region-of-Interest (RoI) identified at L1 or in the full event using algorithms and selections that are close to the ones used in the offline analysis. The HLT selects events, which are stored for offline analysis, at an average rate of $1 \mathrm{kHz}$ during the LHC fill. About 1500 different selections were implemented in the ATLAS trigger system in 2017. This set of selections, the so-called "trigger menu", has been carefully tuned based on the ATLAS physics priorities and the resource usage. A significant accomplishment of the trigger menu in 2017 is that the thresholds on the lepton transverse momentum $\left(p_{\mathrm{T}}\right)$ used when triggering on events with a single electron or muon remained unchanged with respect to 2016, despite the higher peak instantaneous luminosity. More details can be found in Ref. [3].

As part of the improvements for Run-2, the L1 topological trigger (L1Topo) hardware was commissioned in 2016, and is operational since 2017. The L1Topo performs event-topology-based selections using the calorimeter and muon information processed at L1. Examples of such selections are cuts on the angular distance or on the invariant mass of pairs of particle candidates reconstructed at L1. These selections proved to be crucial for triggering on low- $p_{\mathrm{T}}$ signatures at an affordable rate. This is the case for the di- $\tau$ trigger used to select Higgs boson decays into pairs of hadronically decaying $\tau$ leptons. Figure 1 (left) shows the rates as a function of the instantaneous luminosity of di- $\tau$ triggers with different selections [4]. The triggers with the angular selection $\Delta R=\sqrt{\Delta \phi^{2}+\Delta \eta^{2}}<2.9$ implemented at L1Topo have rates significantly lower than the other triggers for the same $H \rightarrow \tau \tau$ signal acceptance. Events with a pair of soft muons, typically used for $B$-physics measurements, also rely on L1Topo-based triggers. In this case, selections on the invariant mass of the two muons and on their angular distance are used to reduce the rate of the accepted events, as shown in Figure 1 (right) [5]. L1Topo is also used to trigger on difficult final states like $q q H(\rightarrow b b)$ or long-lived particles or Higgsino decays into very soft leptons.

Other improvements at L1 deployed in 2017 are in the L1Calo and the L1Muon systems, which process the inputs from the calorimeter and the muon detectors, respectively. At L1Calo, new filter coefficients and noise cuts have been optimised for the high pile-up conditions and the LHC filling schemes used in 2017. This significantly reduced the rates of triggers for hadronic signatures like 

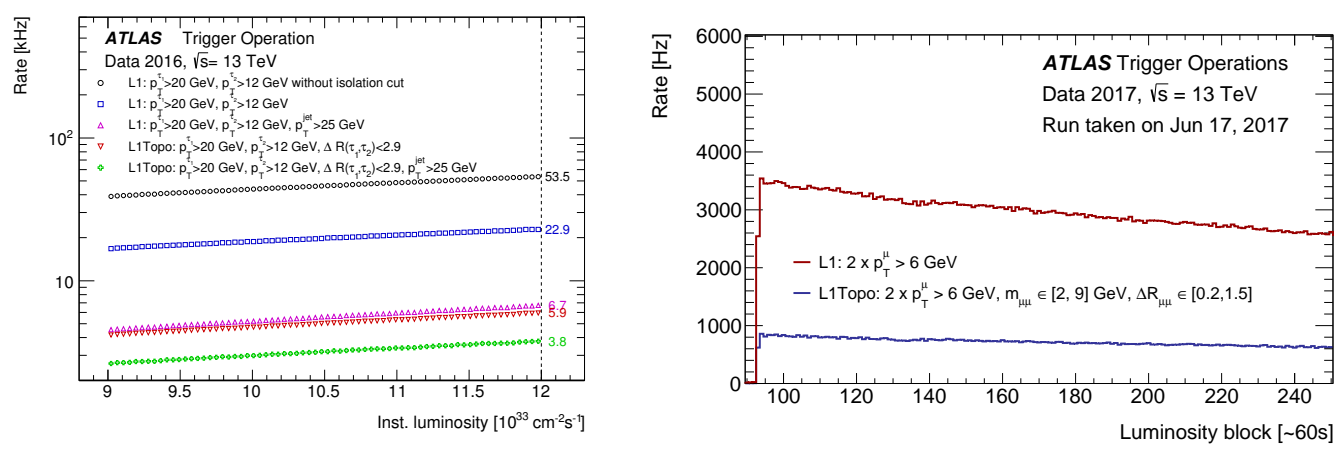

Figure 1: Comparison of L1 rates for triggers with and without selections implemented in L1Topo. Left: rates for di- $\tau$ triggers as a function of the instantaneous luminosity [4]. Right: rates as a function of time in an LHC fill for di-muon triggers [5]. The triggers with L1Topo selections are labelled with "L1Topo".

jets and missing transverse energy $\left(E_{\mathrm{T}}^{\text {miss }}\right)$, and also reduced the non-linear dependence of these rates on the pile-up. It also meant lower calorimeter occupancy and therefore faster processing of the calorimeter information at the HLT. At L1Muon, improved chamber-by-chamber coincidence windows and a new coincidence between the Tile calorimeter and the TGC chambers resulted in a significantly lower rate for the single muon trigger without any noticeable efficiency loss.

At the HLT, in 2017, extensive work has been done in optimising algorithms and selections to reduce the required processing power and better cope with the high pile-up. Efforts were spent in particular on the most CPU-intensive algorithms such as the reconstruction of particle tracks which requires resources that scale non-linearly with pile-up. Other significant developments were deployed for the $E_{\mathrm{T}}^{\text {miss }}$ and jet triggers. The so-called "pufit" algorithm for the $E_{\mathrm{T}}^{\text {miss }}$ calculation was developed to improve the stability of the rates at the HLT versus pile-up. Compared to the "mht" algorithm used in 2016, the "pufit"-based selection yields an HLT rate one order of magnitude lower at $\mu=55$ for the same efficiency [5]. This improvement allowed to keep the threshold of the $E_{\mathrm{T}}^{\text {miss }}$ trigger unchanged from 2016. In the jet trigger, a new jet energy calibration based on both calorimeter and tracking information has been developed to bring the energy measured at the HLT closer to the one measured in offline. The improved calibration yields a steeper efficiency curve so that a higher efficiency and a lower rate can be achieved compared to 2016 [5].

To maximise the physics output, "End-of-Fill" triggers are activated at instantaneous luminosities below $1 \times 10^{34} \mathrm{~cm}^{-2} \mathrm{~s}^{-1}$, when the L1 rate and the usage of the HLT farm are well below their limits. These are triggers too expensive in terms of rate or CPU resources to run at peak luminosity. This is the case for the low- $p_{\mathrm{T}}$ single jet trigger used to collect events for the search for low-mass di-jet resonances [6]. This search is performed using trigger-level objects so that the size of the recorded event is tiny and the HLT output is only marginally increased. Triggers for $B$-physics measurements are also activated as "End-of-Fill" triggers as they are CPU intensive.

Since August 2017, unexpected beam losses forced the LHC to run with a reduced number of bunches in the ring. The compensate for the loss in luminosity, the LHC delivered collisions at the instantaneous luminosity of $2 \times 10^{34} \mathrm{~cm}^{-2} \mathrm{~s}^{-1}$ and a pile-up of about 80 . Despite the improvements mentioned earlier, processing collisions with this level of pile-up was beyond the ca- 

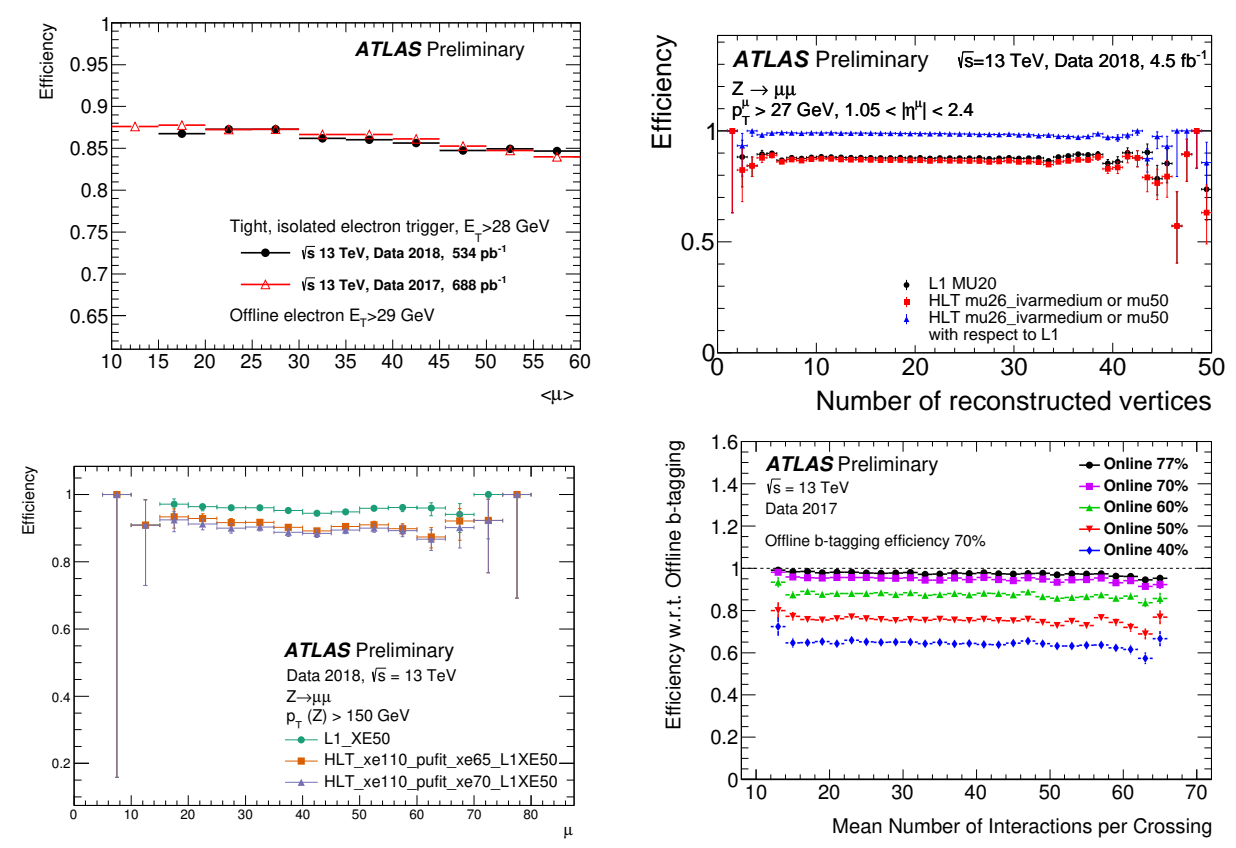

Figure 2: Trigger efficiency as a function of pile-up or number of reconstructed vertices for an isolated electron (top left), isolated muon (top right), $E_{\mathrm{T}}^{\text {miss }}$ (bottom left) and the HLT $b$-jet tagging (bottom right) [5].

pabilities of the HLT farm. ATLAS therefore asked the LHC to run at the "levelled" luminosity of $1.56 \times 10^{34} \mathrm{~cm}^{-2} \mathrm{~s}^{-1}$ and $\mu=60$. This strategy ensured a highly efficient data taking at a marginal loss in integrated luminosity. In 2018, the LHC delivered collisions at instantaneous luminosities of $2 \times 10^{34} \mathrm{~cm}^{-2} \mathrm{~s}^{-1}$ and $\mu=55$ and no levelling has been required so far. After the assessment of the trigger performance at high pile-up in 2017, additional improvements were deployed across all trigger signatures in 2018 to improve the robustness of the trigger efficiency against pile-up. This led to high and stable efficiencies versus pile-up in 2018, as shown in Figure 2 [5].

The ATLAS trigger system maintained excellent data taking efficiency during 2017 and 2018 at record high instantaneous luminosities and pile-up levels. The exploitation of the L1Topo hardware and optimisations of algorithms and selections at the HLT allowed the trigger system to cope with the higher rates and to run a trigger menu largely unchanged from 2016. A successful campaign of optimisation of the selections improved the robustness of the trigger against pile-up.

\section{References}

[1] ATLAS Collaboration, Eur. Phys. J. C 77, 317 (2017)

[2] ATLAS Collaboration, JINST 3, S08003 (2008)

[3] ATLAS Collaboration, ATL-DAQ-PUB-2018-002, https://cds.cern.ch/record/2625986

[4] ATLAS Collaboration, ATLAS-CONF-2017-061, http://cdsweb.cern.ch/record/2274201

[5] ATLAS Collaboration, https://twiki.cern.ch/twiki/bin/view/AtlasPublic/TriggerPublicResults

[6] ATLAS Collaboration, ATL-DAQ-PUB-2017-003, http://cdsweb.cern.ch/record/2295739 